\title{
Martensitic Transformation Development in the Rolled Ti-Ni Alloy: Varying of Temperature Parameters Depending on Grain Orientation
}

\author{
V. Fesenko and Yu. Perlovich \\ Moscow Engineering Physics Institute, Kashirskoe shosse 31, 115409 Moscow, Russia
}

\begin{abstract}
Inhomogeneity of martensitic transformations in the rolled alloy $\mathrm{Ti}-50.3 \% \mathrm{Ni}$ was investigated by use of X-ray texture diffractometry. For the first time temperature parameters of the reverse martensitic transformation $\mathrm{B} 19^{\circ} \rightarrow \mathrm{B} 2$ were measured systematically for grains, corresponding by their orientation to 130 different points within a quadrant of the stereographic projection. Depending on grain orientations, the temperature interval of the reverse martensitic transformation has a width from $20^{\circ}$ to $40^{\circ}$. Obtained data correlate with known regularities of strain hardening distribution in textured materials.
\end{abstract}

\section{INTRODUCTION}

While taking into account an inevitable structure inhomogeneity in metallic materials after any technological treatment, it should be awaited that local temperature parameters of martensitic transformation (MT) would vary by passing from one grain of a polycrystalline specimen to another. According to the X-ray data, obtained by special diffractometric methods, an energy of residual distortion of the crystalline lattice in a rolled metal with developed texture differs for main texture components as well as depends on grain orientation relative to the nearest maximum and minimum on a direct pole figure (DPF) [1]. The content of defects and their arrangement determine temperature stability of resulting structures. If the used heat treatment entails a phase transformation, concrete features of grain substructure would promote or prevent it. depending on mutual correlation of atom displacements, caused by defects or by the change of the initial lattice type [2]. As a rule, a structure inhomogeneity, produced in metallic material by its plastic deformation, can not be removed entirely by the subsequent annealing and gives rise to the structure inhomogeneity of some new type, connected, for example, with inhomogeneous development of recovery processes in a textured polycrystal [3]. Therefore, MT in a real textured sample is the superposition of mutually disagreed MT-processes, localized in grains with different orientations.

An experimental verification of $\mathrm{MT}$-inhomogeneity in a textured polycrystal entails essential difficulties, retarding systematic studies in this field. These difficulties were overcome partially by Liu and Bunge [4], who have shown that MT-processes in a textured $\mathrm{Fe}-30 \% \mathrm{Ni}$ alloy are orientation-dependent. By means of orientation distribution function method (ODF) MT-processes in main texture components were compared and their mutual mismatch was stated. However, emphasis on mathematical formality of the ODF-method restricts possibilities of physical analysis in the course of X-ray measurements.

The present work was aimed to systematic X-ray measurement of MT temperature parameters in a textured $\mathrm{Ti}-\mathrm{Ni}$ alloy and to search for a correlation of MTinhomogeneity with known regularities of strain hardening distribution in deformed metallic materials. In order to split the general MT-process in local subprocesses, X-ray diffractometry was used because of its selective character, allowing to obtain an 
information about grains with crystallografic orientations of interest. Under the standard geometry of diffractometric measurement by each position of the sample in the holder of texture unit only grains with the definite orientation are reflecting $X$-rays,- just this principle is in the basis of texture diffractometry [5]. MT is accompanied by an intensity increase for $\mathrm{X}$-ray lines of the new phase, testifying about appearance of its grains with the registered orientation. Then the challenge was to cause MT in the sample. mounted on the texture diffractometric unit, and to compare systematically parameters of MT in grains with different orientations, corresponding to different points of some PF.

\section{MATERIAL AND PROCESSING}

For investigation the alloy $\mathrm{Ti}-50.3 \% \mathrm{Ni}$ was choosed, known by its good shape memory properties [6]. Under cooling in the alloy $\mathrm{Ti}-50.3 \% \mathrm{Ni}$ the direct MT (DMT) $\mathrm{B} 2 \rightarrow \mathrm{B} 19^{\prime}$ takes place in the temperature interval $40^{\circ}-15^{\circ} \mathrm{C}$, while under heating the reverse MT (RMT) $\mathrm{B} 19^{\circ} \rightarrow \mathrm{B} 2$ - in the interval $50^{\circ}-80^{\circ} \mathrm{C}$. The phase $\mathrm{B} 2$ has the $\mathrm{BCC}$ crystalline lattice of $\mathrm{CsCl}$ type and the phase $\mathrm{B}^{\prime} 9^{\prime}$ has the rhombic lattice with an additional monoclinic distortion.

The alloy was rolled at $700^{\circ}-800^{\circ} \mathrm{C}$ down to thickness of $0.6 \mathrm{~mm}$. Obtained sheet was cut into specimens $20 \times 20 \mathrm{~mm}$ in size. X-ray phase analysis of specimens shows, that at room temperature in consequence of the partial DMT they contain both phases, but the phase $\mathrm{B}^{\prime} 9^{\prime}$ is predominant.

\section{METHOD OF INVESTIGATION}

The idea of the experiment consists in continuous measuring of $\mathrm{X}$-ray line intensity for grains with a definite orientation during heating (cooling) of a specimen on the texture unit. As the phase B19' transforms into the phase B2, intensity of B2-reflections increases. and kinetics of this increase corresponds to development of RMT. Comparison of such RMT-curves, obtained for grains with different orientations, would allow to estimate the regular inhomogeneity of RMT.

For realization of this idea the special holder of specimens was made and set on the texture unit of the X-ray diffractometer DRON-3. On the interior cylindrical surface of this holder the controlled heater was installed, allowing to rise temperature of the specimen in the linear regime up to $200^{\circ} \mathrm{C}$. The chromel-alumel thermocouple measured temperature at the back surface of the specimen, which was pressed closely against the holder body to avoid an essential temperature gradient. The rate of specimen heating by the optimal regime was equal to $20 \mathrm{deg} / \mathrm{min}$. The two-coordinate recorder INDIM-5 was used for registration of X-ray line integral intensity versus temperature of the specimen.

A typical curve of intensity increase by RMT $\mathrm{B} 19^{\prime} \rightarrow \mathrm{B} 2$ for the $\mathrm{X}$-ray line $(011)_{\mathrm{B} 2}$ is shown on Fig.1-a. Though DMT was partial and the specimen contains both phases at room temperature, due to existance of the thermal hysteresis the start temperature $A_{S}$ of RMT can be achieved only as a result of some heating. The following parameters of RMT-curves were measured:

$$
\begin{aligned}
& \mathrm{I}_{\max }-\text { integral intensity of the } \mathrm{X} \text {-ray line }(011)_{\mathrm{B} 2} \text { at a temperature exceeding } \\
& \text { the final temperature } \mathrm{A}_{\mathrm{f}} \text { of } \mathrm{RMT} \text {; } \\
& \mathrm{T}_{0.1}, \mathrm{~T}_{0.5}, \mathrm{~T}_{0.9} \text { - temperatures, corresponding to attainment of } 0.1 \mathrm{I}_{\max } \\
& \quad 0.5 \mathrm{I}_{\max } \text { and } 0.9 \mathrm{I}_{\max } \text { respectively; } \\
& \Delta \mathrm{T}=\mathrm{T}_{0.9}-\mathrm{T}_{0.1} .
\end{aligned}
$$

By presentation of obtained experimental data $T_{0.5}$ and. $\mathrm{T}$ are used more often, as a middle and a width of RMT temperature interval. It is difficult to determine in practice temperatures $A_{\mathrm{s}}$ and $A_{f}$ by RMT-curves with satisfactory precision; therefore, instead of these accurate limits of RMT-interval, $\mathrm{T}_{0.1}$ and $\mathrm{T}_{0.9}$ were determined. As a result, $\Delta \mathrm{T}$ is somewhat narrower than the real temperature interval of RMT. 
Since measurement of RMT-curves for different orientations of reflecting B2 grains requires multiple heating of the same specimen, it is necessary to be sure, that previous thermal cycles have no effect on measured MT parameters. Such situation could be attained. when a number of previous cycles would be sufficiently great, causing stabilization of MT parameters [6]. In order to determine this number for the rolled alloy of interest, the dependence of MT parameters on number of thermal cycles, connected both with RMT and DMT, was studied.

Thermal cycling was realized by use of specimen heating up to $150^{\circ} \mathrm{C}$ in the drying case and its subsequent cooling in liquid nitrogen. In the initial state of the specimen and after each fifty eycles the X-ray curve for RMT was registered at the same position of the specimen on the texture unit. Results of these measurements are shown on Figure 1b. $T_{0.1}$ and $T_{0.5}$ cease to change after first 50 thermal cycles already. Therefore, rolled specimens, destined for systematic study of MT inhomogeneity, were put beforehand through 50 thermal cycles.

The geometry of diffractometric texture measurements was used by recording of RMT-curves. while the specimen was brought successively in different positions, corresponding to registration of the same X-ray reflection for B2-grains with different crystallographic orientations. Behind this procedure is the standard diffractometric method of direct pole figures [5]. For texture analysis the $X$-ray line $\{011\} \mathrm{B} 2$ was chosen, because it is the most intense and does not overlap with lines of the phase B19'.

The rolling texture of the phase $\mathrm{B} 2$, measured at $150^{\circ} \mathrm{C}$ (Fig.2-a), contains components of different types: a maximum of the component with a rolling plane $\{011\}$ is located in the center of $\operatorname{DPF}\{011\}$, while components $\{111\}<112>$ form a joint extended maximum along the circle with an angular radius of $35^{\circ}$. The same DPF $\{011\}$. measured after cooling of the specimen down to $35^{\circ} \mathrm{C}$ (Fig.2-b), differs sharply from the initial one due to relative weakening of components $\{111\}<112>$. Hence. these components experience DMT by specimen cooling first of all. Such difference in behaviour of main texture components in the course of MT is a convincing starting point for detailed further measurements of MT inhomogeneity.

\section{RESULTS AND DISCUSSION}

Curves of RMT were measured for 130 points, placed within limits of the quadrant on the partial DPF $\{011\} \mathrm{B}_{2}$. Values of $\mathrm{T}_{0.5}$ and $\Delta \mathrm{T}$ were plotted on a stereographic projection and their distributions were found to be rather smooth and ordered. Contours of equal MT parameters were drawn (Fig.3-a,b), and obtained diagrams were compared with the distribution of pole density in the corresponding quadrant of DPF. In order to check, whether location of maxima and minima on these diagrams is regular or occasional. similar measurements were fulfilled additionally for some DPF regions of several other specimens, which had been cut out from the initial rolled sheet. All these specimens had the same rolling texture with an accuracy of inevitable texture inhomogeneity. Correspondingly, principal features of distributions, shown on Fig.3, are unchanged by passing from one specimen to another.

These features are following:

a) depending on grain orientations, $T_{0.5}$ varies within a temperature interval of $8^{\circ}$ width, while $\Delta \mathrm{T}$ takes values from $20^{\circ}$ to $40^{\circ}$;

b) near the longitudinal cross section of the sheet, containing its rolling and normal directions, the zone of increased RMT parameters is located;

c) both $T_{0.5}$ and $\Delta T$ attain their minimal values in the central part of the texture maximum, corresponding to the component $\{111\}<112>$;

d) temperature parameters of RMT increase at periphery regions of texture maxima;

e) the texture maximum, belonging to the component with the rolling plane $\{011\}$ and situated at the center of DPF $\{011\}$, falls in the DPF region, where superposition of tendencies b) and d) takes place;

f) development of RMT seems to be anisotropic in grains of the same texture component, i.e. formation of the phase B2 has different kinetics being observed by use of $\mathrm{X}$-ray diffraction along different directions in the same grains.

The temperature interval of RMT, measured by the used X-ray method, is somewhat wider, than the method of electrical resistivity has shown. The latter gives an integral 

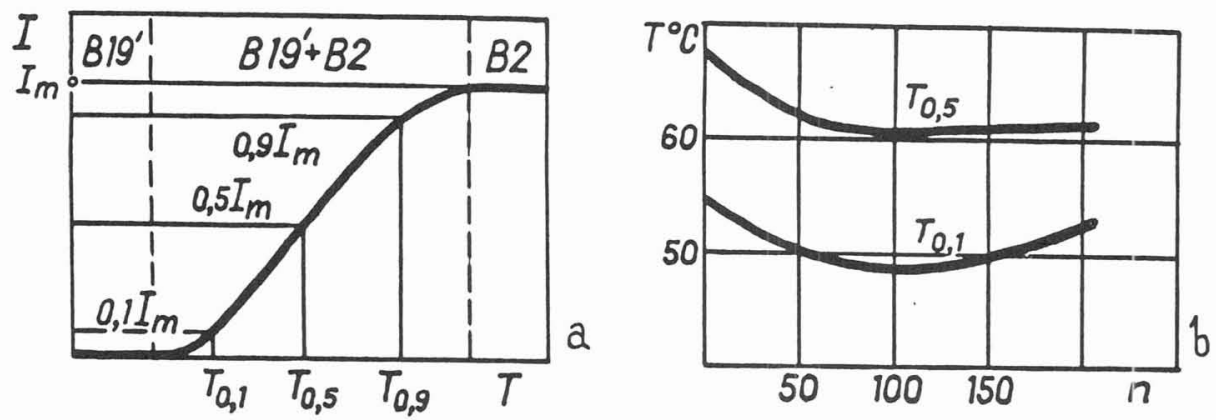

Higure 1: a) curves of intensity increace by RMT B19 $\rightarrow B 2$;

b) influence of thermal cycling on $T_{0.1}$ and $T_{0 .}$ of RMT.
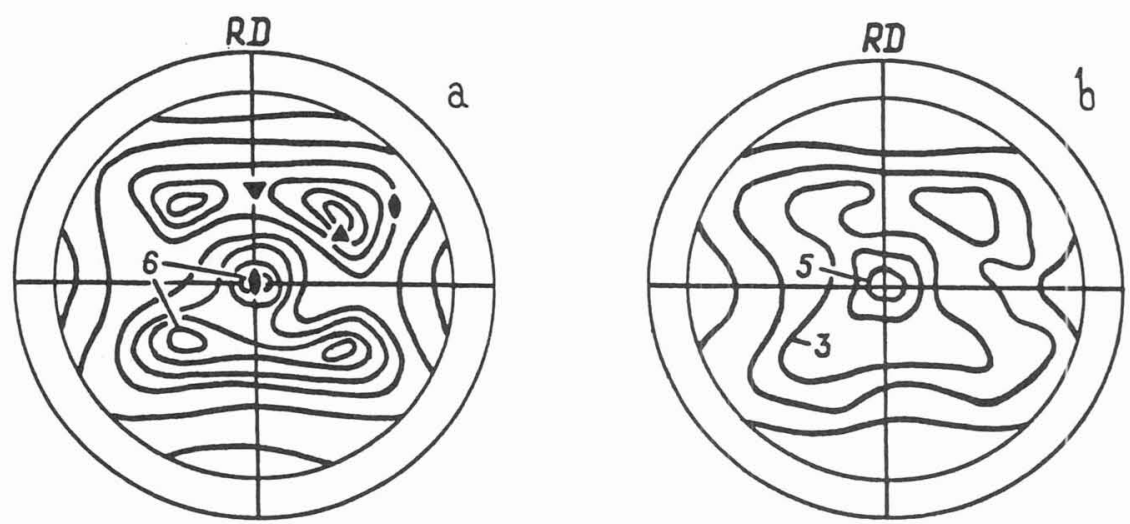

Figure 2: DPF $\{011\}$ Ba, measured at $150^{\circ} \mathrm{C}$ (a) and $35^{\circ} \mathrm{C} \mathrm{(b)}$.

Heights of texture maximums are indicated in units of registered intensity. $\quad \cdot\{011\}<011>, \Delta, \nabla \cdot\{111\}<112>$.

RD

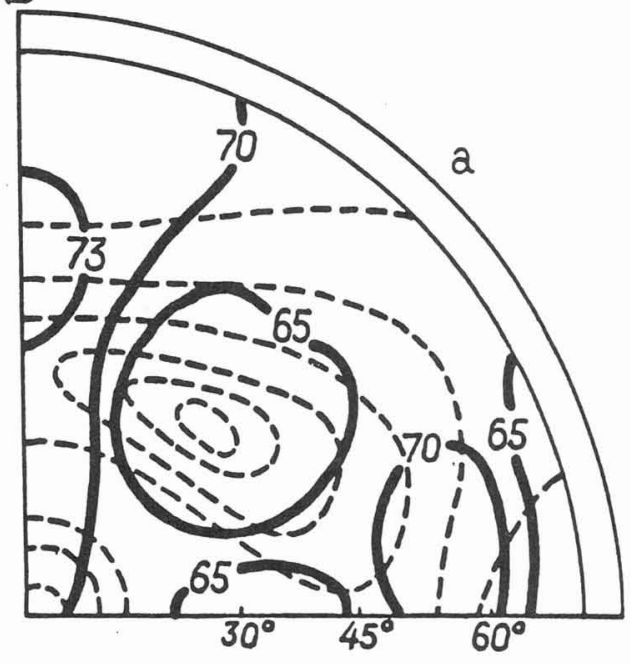

$R D$

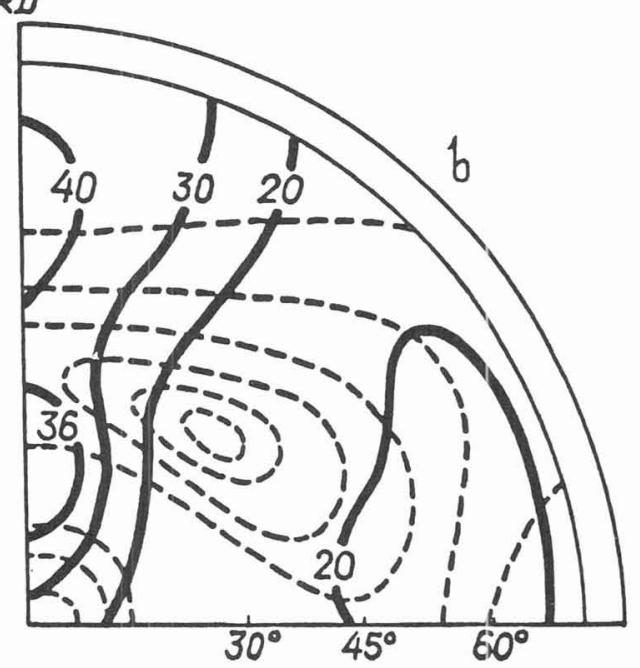

Figure 3: Distributions of Tos (a) and $\Delta T$ (b) for RMT B19 $\rightarrow B 2$. Texture contours are shown with dotted lines. 
estimation and is insensitive to local variations of MT-parameters in grains with a small relative volume. At the same time the above-mentioned selective character of $\mathrm{X}$-ray measurements promotes an extreme rise of their sensitivity.

Obtained results agree with general concepts, concerning development of a regular structure inhomogeneity in textured polycrystals, i.e. the distinct correlation between substructure features of grains and their crystallographic orientation $[1,3]$. In particular, it was shown for rolled BCC-metals, that grain dispersity and lattice distortion increase in going from the central part of texture maximum to its periphery regions. This principle has universal character, being a result of processes responsible for texture formation and stability of final grain orientations. According to points c) and d) of the foregoing list, primarily RMT develops and finishes in grains, whose crystallographic orientations correspond to the central part of the texture maximum, while at its periphery RMT is retarded and stretched by the temperature scale (Fig.1-a). Comparison of DPF $\{011\}_{\mathrm{B} 2}$, measured at $150^{\circ}$ and $35^{\circ} \mathrm{C}$ (Fig.2), as well as diagrams of RMTparameters (Fig.3) testify that the higher is the temperature of DMT in B2-grains with some orientations by cooling of the specimen, the lower is the temperature of RMT in B19'-grains with corresponding orientations by subsequent heating. Hence, the width of thermal hysteresis is maximal for grains with orientations in the central part of the texture maximum $\{111\}<112>$. Namely in these grains both DMT and RMT develop most easily.

At least two important conclusions can be made on this basis. Firstly, some obstacles prevent RMT in grains, corresponding to periphery regions of the texture maximum; evidently. above-mentioned grain dispersity and lattice distortion play in these regions the role of such obstacles. Secondly, in the course of DMT B2 $\rightarrow$ B19' a structure inhomogeneity of the initial deformed phase $\mathrm{B} 2$ is inherited by the derivative phase B19', and in a similar way by RMT B19' $\rightarrow$ B2 new grains of the phase B2 acquire substructure features of the phase $\mathrm{B}^{\circ} 9^{\prime}$, inherited earlier from the primary phase $\mathrm{B} 2,-$ this is obvious from inhomogeneous distributions of RMT temperature parameters (Fig.3).

The field of residual stresses has an influence on MT development also. Namely this field is responsible for an increase of RMT-parameters near the longitudinal cross section of the sheet. A question remains, whether such stress-induced features of RMTinhomogeneity depend on the type of a texture, formed in the alloy by its rolling. In any case, control of texture formation in the course of rolling and heat treatment would allow to have an effect on temperature parameters of MT in the rolled polycrystalline alloy. In particular, the sharper would be a texture, the narrower would be the MTinterval.

\section{CONCLUSIONS}

1. With reference to the textured alloy $\mathrm{Ti}-20 \% \mathrm{Ni}$ the $\mathrm{X}$-ray method was elaborated to study MT kinetics in grains with crystallographic orientations of interest.

2. For the first time distributions of MT temperature parameters were constructed for a textured polycrystal, testifying about essential inhomogeneity of the investigated process.

3. MT in the rolled alloy develops in accordance with structure inhomogeneity, typical for deformed metallic materials: MT meets the greatest amount of retarding obstacles at. periphery regions of texture maxima, where (sub)grains are most disperse and/or their crystalline lattice is the most distorted.

4. Initial structure inhomogeneity of the phase B2. caused by deformation. is inherited by derivative phases and retains by repeated MT.

5. An influence of residual stresses on MT inhomogeneity superposes on texture effects. 


\section{REFERENCES}

[1] Perlovich Yu., "Development of strain hardening inhomogeneity during texture formation under rolling of bcc-metals". Numerical Prediction of Deformation Processes and the Behaviour of Real Materials, 15th Riso International Symposium on Material Science, 5-9 September 1994, S.I.Andersen et al. Eds. (Riso National Laboratory, Roskilde. Denmark, 1994) pp.445-450.

[2] Bockstein S.Z., Structure and properties of metal alloys (Publishing House "Metallurgiya". Moscow, 1971) pp.252-278 (in Russian).

[3] Perlovich Yu. "Inhomogeneity of strain hardening, recovery and recrystallization in molybdenum alloys with a developed crystallographic texture", Structure, Texture and Mechanical Properties of Deformed Molybdenum. V.I.Trefilov Ed. (Publishing House "Naukova Durnka", Kiev, 1983) pp.88-145 (in Russian).

[4] Liu W.P. and Bunge H.J., "The orientation-dependent characteristics of martensitic transformation in cold-rolled $\mathrm{Fe}-30 \% \mathrm{Ni}$ alloy", Eighth International Conference on Textures of Materials (ICOTOM 8). J.S.Kallend and G.Gottstein Eds. (The Metallurgical Society, 1988) pp.755-761.

[5] Rusakov A.A., X-raying of metals (Publishing House "Atomizdat", Moscow, 1977) pp.345-365 (in Russian).

[6] Alloys with shape memory effect, H.Funakubo Ed. (Publishing House "Metallurgiya". 1990) pp.58-98 (in Russian). 